\title{
Comportamento higroscópico da polpa de Araçá-boi (Eugenia stipitata) em pó obtida pelo método de liofilização com diferentes concentrações de maltodextrina
}

\author{
Higroscopic behavior of Araçá-boi (Eugenia Stipitata) powder obtained by the lyophilization
}

method with different maltodextrin concentrations

Comportamiento higroscópico del polvo de Araçá-boi (Eugenia Stipitata) obtenido por el método de liofilización con diferentes concentraciones de maltodextrina

Recebido: 25/05/2021 | Revisado: 31/05/2021 | Aceito: 16/07/2021 | Publicado: 25/07/2021

Romário Oliveira de Andrade ORCID: https://orcid.org/0000-0001-8377-093X

Instituto Federal de Alagoas, Brasil

E-mail: romario.andrade@ifal.edu.br

Nadson Libio Bezerra Ferreira

ORCID: https://orcid.org/0000-0003-0190-6034

Universidade Estadual de Campinas, Brasi

E-mail: pesq.nadson@gmail.com

Gustavo Santos de Lima

ORCID: https://orcid.org/0000-0002-7280-0838 Universidade Federal de Campina Grande, Brasil

E-mail: gustavosantosdelima@gmail.com

Ana Raquel Carmo de Lima ORCID: https://orcid.org/0000-0002-6237-3553 Universidade Federal de Campina Grande, Brasil

E-mail: anakel_alimentos@hotmail.com

Caio Franklin Vieira de Figueiredo ORCID: https://orcid.org/0000-0003-0364-164X Universidade Federal de Campina Grande, Brasil

E-mail: caiovieirafigueiredo@gmail.com

Eduardo Henrique Santos de Lima

ORCID: https://orcid.org/0000-0002-9147-3940 Universidade Federal de Campina Grande, Brasil

E-mail: eduardohsantosdelima@ gmail.com

Saul Ramos de Oliveira

ORCID: https://orcid.org/0000-0002-2804-6671 Universidade Federal da Paraíba, Brasil

E-mail: sauloramos86@gmail.com

Edilayane da Nóbrega Santos

ORCID: https://orcid.org/0000-0002-9551-6442

Universidade Federal de Lavras, Brasil

E-mail: edilayane.santos@estudante.ufla.br

Josivanda Palmeira Gomes

ORCID: https://orcid.org/0000-0002-2047-986X

Universidade Federal de Campina Grande, Brasil E-mail: josivanda@gmail.com

Ana Paula Trindade Rocha

ORCID: https://orcid.org/0000-0002-0230-7394

Universidade Federal de Campina Grande, Brasil E-mail: ana_trindade@yahoo.com.br

Gilsandro Alves da Costa

ORCID: https://orcid.org/0000-0001-6562-0022 Universidade Federal da Paraíba, Brasil

E-mail: gilsandrocosta@gmail.com

Anderson Ferreira Vilela

ORCID: https://orcid.org/0000-0001-8034-090X

Universidade Federal da Paraíba, Brasil

E-mail: prof.ufpb.anderson@gmail.com

Resumo

Esse estudo objetivou-se avaliar as características físico-químicas do pó de Araçá-boi liofilizado, e o comportamento higroscópico utilizando isotermas de adsorção e diferentes modelos matemáticos. As formulações da polpa de Araçáboi com diferentes concentrações de maltodextrina $(14,21$ e $28 \%)$ foram congeladas em freezer à $-18^{\circ} \mathrm{C}$, em seguida, 
desidratadas em liofilizador de bancada. Foram determinados os parâmetros de teor de água, sólidos totais, $\mathrm{pH}$ e acidez titulavel. As isotermas de adsorção de água do pó de Araçá-boi nas temperaturas de 25,30 e $35{ }^{\circ} \mathrm{C}$ foram determinadas usando o método especial indireto estático, utilizando o higrômetro Aqualab, para medir a atividade de água. Os modelos de Henderson, Oswin, Halsey, GAB e Peleg foram ajustados às isotermas de adsorção de água utilizando regressão não linear. A partir do coeficiente de determinação $\left(\mathrm{R}^{2}\right)$ e do desvio percentual médio $(\mathrm{P})$, verificou-se que, entre os modelos estudados e considerando as temperaturas testadas, os valores de $R^{2}$ obtidos em GAB, Halsey e Peleg estão tão próximos entre si que, no pior dos casos, não superam 0,9\% de diferença. A melhor representação matemática das isotermas foi obtida pelo modelo de $\mathrm{GAB}$, uma vez que apresentou os menores valores para $\mathrm{X}_{\mathrm{m}}$, sendo, portanto, o modelo que melhor explica o ganho de água.

Palavras-chave: Eugenia stipitata; Isotermas; Modelos matemáticos; Atividade de água; Estabilidade microbiológica.

\begin{abstract}
This study aimed to evaluate the physicochemical characteristics of freeze-dried Araçá-boi powder, and its hygroscopic behavior using adsorption isotherms and different mathematical models. The Araçá-boi pulp formulations with different concentrations of maltodextrin $\left(14,21\right.$ and $28 \%$ ) were frozen in a freezer at $-18{ }^{\circ} \mathrm{C}$, then dehydrated in a bench-top freeze dryer. The parameters of water content, total solids, $\mathrm{pH}$ and titratable acidity were determined. The water adsorption isotherms of Araçá-boi powder at temperatures of 25,30 and $35{ }^{\circ} \mathrm{C}$ were determined using the special indirect static method, using the Aqualab hygrometer, to measure water activity. The Henderson, Oswin, Halsey, GAB and Peleg models were fitted to the water adsorption isotherms using nonlinear regression. From the coefficient of determination $\left(\mathrm{R}^{2}\right)$ and the mean percentage deviation $(\mathrm{P})$, it was found that, among the models studied and considering the temperatures tested, the $\mathrm{R}^{2}$ values obtained in GAB, Halsey and Peleg are so close to each other that, in the worst case, they do not exceed a $0.9 \%$ difference. The best mathematical representation of the isotherms was obtained by the GAB model, since it presented the lowest values for $\mathrm{X}_{\mathrm{m}}$, being, therefore, the model that best explains the water gain.
\end{abstract}

Keywords: Eugenia stipitata; Isotherms; Mathematical models; Water activity; Microbiological stability.

\title{
Resumen
}

Este estudio tuvo como objetivo evaluar las características fisicoquímicas del polvo de Araçá-boi liofilizado y su comportamiento higroscópico utilizando isotermas de adsorción y diferentes modelos matemáticos. Las formulaciones de pulpa de Araçá-boi con diferentes concentraciones de maltodextrina (14, 21 y 28\%) se congelaron en un congelador a $-18^{\circ} \mathrm{C}$ y luego se deshidrataron en un liofilizador de mesa. Se determinaron los parámetros de contenido de agua, sólidos totales, $\mathrm{pH}$ y acidez titulable. Las isotermas de adsorción de agua del polvo de Araçá-boi a temperaturas de 25,30 y $35^{\circ} \mathrm{C}$ se determinaron utilizando el método estático indirecto especial, utilizando el higrómetro Aqualab, para medir la actividad del agua. Los modelos de Henderson, Oswin, Halsey, GAB y Peleg se ajustaron a las isotermas de adsorción de agua mediante regresión no lineal. A partir del coeficiente de determinación $\left(\mathrm{R}^{2}\right)$ y la desviación porcentual media $(\mathrm{P})$, se encontró que, entre los modelos estudiados y considerando las temperaturas probadas, los valores de $\mathrm{R}^{2}$ obtenidos en GAB, Halsey y Peleg son tan cercanos entre sí. que, en el peor de los casos, no superen el $0,9 \%$ de diferencia. La mejor representación matemática de las isotermas se obtuvo mediante el modelo GAB, ya que presentó los valores más bajos para Xm, siendo, por tanto, el modelo que mejor explica la ganancia de agua.

Palabras clave: Eugenia stipitata; Isotermas; Modelos matemáticos; Actividad del agua; Estabilidad microbiológica.

\section{Introdução}

O araçá-boi (Eugenia stipitata) pertencente à família Myrtaceae, é uma fruta que se apresenta naturalmente nas regiões contempladas pela Floresta Amazônica, abrangendo países como Peru, Bolívia, Equador, Colômbia e Brasil, a qual também vem sendo cultivado em regiões como sul da Bahia (De Souza et al., 2018). Essa fruta ainda tem sua disseminação moderadamente retraída para outras regiões, principalmente pelas suas características de alta perecibilidade no seu estado in natura (Iturri et al., 2021).

No quesito sensorial, a fruta se destaca possuindo uma polpa carnuda, suculenta, aromática e levemente ácida (W. K. Costa et al., 2020), características comuns em frutos tropicais e muito apreciado tanto para consumo in natura quanto para preparo de sucos e doces.

Além dessas características sensoriais atrativas, outros autores evidenciam o potencial fornecimento de compostos bioativos para essa fruta, Neves et al., (2015), apresentam uma quantidade de conteúdo fenólico total maiores que caju, cajá, inajá e uxi, já Gonçalves et al., (2010) aponta uma capacidade de eliminação radical maior que o tamarindo, maracujá, 
graviola, buriti, cupuaçu e bacuri, outras frutas nativas da Floresta Amazônica. Soares et al., (2019) constatou que a Eugenia stipitata não exerceu efeitos tóxicos nas larvas em nenhuma das doses testada e Neri-Numa et al., (2013) apontaram que o extrato etanólico da polpa de Eugenia stipitata apresentou propriedades antimutagênicas e antigenotóxicas, sendo um potencial preventor de câncer.

Esses conteúdos de compostos químicos, junto de suas qualidades sensoriais e a rusticidade no cultivo que Eugenia stipitata apresenta, a coloca como uma fonte agrícola rica em componentes que possam ser aproveitados na indústria alimentícia e farmacêutica (Calvi et al., 2017).

No entanto apesar de todas essas propriedades o araçá-boi ainda apresenta uma perecibilidade alta, dificultando principalmente a logística no que diz respeito a sua comercialização, além de apresentar poucos estudos relacionados a sua caracterização físico-química e sensibilidade microbiologia o que dificulta ainda mais o conhecimento abrangente de suas características de conservação (Baldini et al., 2017). Com isso faz-se necessário a busca de tecnologias de conservação para manutenção das propriedades funcionais e nutricionais, possibilitando o alcance de novos mercados.

Dentro dessas tecnologias que podem ser aplicadas visando a melhoria na conservação e consequentemente na comercialização do araçá-boi, a liofilização apresenta propriedades que se aplica diretamente a funcionalidade e manutenção de características nutricionais e tecnológicas de diversos produtos, é um método de secagem que utiliza a sublimação para mudança de fases, ou seja, o alimento é primeiro congelado e depois submetido a alto vácuo, por meio do qual o gelo de água evapora sem derreter, isso faz com que principalmente os macro e micro nutrientes tenham o mínimo de alterações estruturais (Berk, 2018).

Orqueda et al., (2017), ao avaliarem o pó da polpa de Ziziphus mistol argentino liofilizado, constaram uma presença de fenólicos totais de 356,0 mg GAE/100g, valores muito próximos e até maiores do que em frutas in natura reconhecidas pelo seu potencial fenólico como uvas, com uma média de $425 \mathrm{mg} \mathrm{GAE} / 100 \mathrm{~g}$ (Miletić et al., 2014), figos com média de $195 \mathrm{mg}$ GAE/100g (Najafabadi et al., 2017) e frutas jujubas 565 mg GAE/100g (Sun et al., 2011), esses estudos mostram a eficiência da liofilização na integridade desses compostos fenólicos.

Além da composição fenólica características sensoriais e físicas também são largamente conservadas quando utilizado a liofilização, a casca da romã liofilizada apresentou valores de cor para $\mathrm{L}^{*}, \mathrm{a}^{*} \mathrm{e} \mathrm{b}^{*}$ maiores que as cascas secas em gabinete mecânico, isso pode ser explicado pela alta temperatura utilizada na secagem com gabinete o que pode levar ao escurecimento do flavedo, diminuindo desta forma o brilho de cor avermelhada presente na casca da romã, muito atrativo sensorialmente (Hamid et al., 2020).

No entanto, essas técnicas de conservação e processamento de alimentos por mais tecnológicas e práticas que sejam, modificam as características estruturais dos alimentos, mesmo que em escala nanométrica, isso por que os alimentos em geral possuem propriedades heterogênicas, porosas e higroscópicas simultaneamente (Karim et al., 2018). A higroscopicidade é a capacidade que o alimento em pó apresenta de absorver e capturar a umidade do ar, ou seja, a capacidade de retenção de água do ambiente (Çopur et al., 2019).

Para entender melhor a propriedade de higroscopicidade em alimentos em pó, são utilizados modelos matemáticos os quais avaliam o comportamento das isotermas de sorção, em bora ainda não exista uma equação geral que descreva o comportamento dessas isotermas, principalmente pela diversidade do conteúdo dos alimentos, que afeta diretamente na atividade de água (G. S. Oliveira et al., 2014), a avaliação do comportamento dessas isotermas contribui para ideal escolha do tempo de secagem, embalagens a serem utilizadas, determinação na caracterização do produto, influenciando diretamente na estabilidade e vida útil (Cavalcante et al., 2018).

Praticamente todas as frutas secas apresentam uma grande quantidade de açúcares concentrados após a liofilização, as quais atribuem um estado amorfo a esses alimentos, e consequentemente uma alta higroscopicidade, sendo prejudicial a 
estabilidades de armazenamento, processamento e controle da textura desses pós (Djendoubi Mrad et al., 2012). Para diminuir essa higroscopicidade adjuvantes podem ser adicionados a esses pós. A maltodextrina é um adjuvante muito utilizado na obtenção de alimentos em pó, podendo diminuir a aglomeração nesses alimentos melhorando sua fluidez, sua utilização se destaca principalmente pela sua alta $T_{g}$ (temperatura de transição vítrea), além de possuir sabor suave e baixo preço (Fongin et al., 2019; Palzer \& Sommer, 2011).

Com isso, o objetivo deste estudo foi avaliar as características físico-químicas e microbiológicas do pó de araçá-boi liofilizado com diferentes concentrações de maltodextrina, e verificar o seu comportamento higroscópico através das isotermas de adsorção utilizando diferentes modelos matemáticos.

\section{Metodologia}

\subsection{Matéria-prima}

O estudo realizado apresenta natureza quantitativa (Pereira et al., 2018). Os frutos de araçá-boi (Eugenia stipitata) foram adquiridos na Fazenda Amizade, Vila Brasil, situada na cidade de Una/BA, ainda em seu estado in natura. Os frutos foram selecionados de acordo com seu estado de maturação adequado, sendo descartados os que não se encontravam dentro dos padrões. Logo após essa seleção os frutos foram acondicionados em caixas térmicas e transportados para o Laboratório de Armazenamento e Processamento de Produtos Agrícola, da Universidade Federal de Campina Grande, PB. O processo de sanitização dos frutos foi realizado utilizando hipoclorito de sódio a $100 \mathrm{ppm}$ por $10 \mathrm{~min}$, sendo enxaguados e lavados em seguida. Posteriormente os frutos foram despolpados, utilizando despolpadeira horizontal com peneira de $0,8 \mathrm{~mm}$ de diâmetro. A polpa foi acondicionada em sacos plásticos de polietileno com $100 \mathrm{~g}$ cada um, e armazenados em freezer congelados a -18 ${ }^{\circ} \mathrm{C}$, até o momento das análises.

\subsection{Formulações}

As formulações foram divididas de acordo a porcentagem de adição de maltodextrina sendo elas com 14, 21 e $28 \%$, após essa adição as polpas foram homogeneizadas e embaladas em formas plásticas e acondicionadas em freezer horizontal sob congelamento a $-18^{\circ} \mathrm{C}$ por 48 horas.

\subsection{Liofilização}

Após congelamento total as amostras foram transferidas para Liofilizador de bancada Christ, modelo ALPHA 1-2 LDplus, Martin Christ Gefriertrocknungsanlagen, Osterode em Harz, Alemanha, refrigerado a uma temperatura de $-40^{\circ} \mathrm{C}$ durante 48 horas. Após secagem o material seco de cada amostra foi triturado usando almofariz e pulverizados usando misturador de bancada.

\subsection{Análises físico-químicas}

Todas as análises foram realizadas em triplicata, conforme os procedimentos analíticos do Instituto Adolfo Lutz (IAL, 2008), onde foram avaliados o teor de água e sólidos totais pelo método de secagem das amostras em estufa à $105^{\circ} \mathrm{C}$, até peso constante; $\mathrm{pH}$ determinado na amostra diluída em água destilada, utilizando pHmetro digital; a acidez total titulável por titulometria com $\mathrm{NaOH} 0,1 \mathrm{~N}$; os sólidos solúveis totais foram determinados através de leitura direta da amostra em refratômetro portátil.

Para o teor de ácido ascórbico foi utilizada a metodologia da AOAC, (2016), onde foi utilizada o método de determinação através da titulação com o 2,6-diclorofenolindofenol sódio (DCFI) até obtenção da coloração rosa claro. A medida da atividade de água (aw) foi realizada por meio do equipamento Aqualab modelo 3TE (Decagon Devices, Inc.). 
A determinação da cor das amostras foi realizada obtendo-se os parâmetros L*, a* e b* medidos com espectrofotômetro portátil Hunter Lab Mini Scan XE Plus, em que L* define a luminosidade $\left(\mathrm{L}^{*}=0-\right.$ preto e $\mathrm{L}^{*}=100-$ branco) e $\mathrm{a}^{*}$ e b* são responsáveis pela cromaticidade (+a* vermelho e $-\mathrm{a}^{*}$ verde; $+\mathrm{b}^{*}$ amarelo e $-\mathrm{b}^{*}$ azul).

A partir dos valores de $a^{*}$ e $b^{*}$, os valores de croma $\left(C^{*}\right)$ e ângulo de tonalidade foram calculados seguindo (Equação 1) e (Equação 2) respectivamente.

$$
\begin{aligned}
& C^{*}=\sqrt{\left(a^{*}\right)^{2}+\left(b^{*}\right)^{2}} \\
& h=\tan ^{-1}\left(\frac{a^{*}}{b^{*}}\right)
\end{aligned}
$$

Para análise de solubilidade foi seguido a metodologia descrita por Eastman \& Moore, 1984, (1984) com adaptações de Cano-Chauca et al., (2005) e Botrel et al., (2016). Primeiramente se adicionou 1g de pó do araçá-boi, em 100ml de água destilada e agitado durante 5 minutos em agitador magnético, o pó diluído na água destilada foi centrifugado a uma rotação de $2600 \mathrm{rpm}$ durante 5 minutos. Em seguida uma alíquota de $25 \mathrm{ml}$ do sobrenadante foi transferida para uma placa de Petri, pesada e submetida à secagem em estufa a $105^{\circ} \mathrm{C}$ por 24 horas. A Equação 3 foi utilizada para calcular a solubilidade.

$$
S=\left[\left(\frac{M s}{M a}\right) \times 4\right] \times 100
$$

Onde:

$\mathrm{S}=$ solubilidade $(\%)$

Ms = massa dos sólidos dissolvidos no sobrenadante $(\mathrm{g}) ; \mathrm{e}$,

$\mathrm{Ma}=$ massa da $\operatorname{amostra}(\mathrm{g})$.

Para a avaliação da molhabilidade utilizou-se a metodologia descrita por Freudig et al., (1999) com adaptações de Vissotto et al., (2010). Foi adicionado de maneira suave $1 \mathrm{~g}$ da amostra em $100 \mathrm{ml}$ de água destilada á $25^{\circ} \mathrm{C}$ e determinado visualmente o tempo necessário para que todas as partículas se molhassem. Com isso utilizando a Equação 4 foi calculado a taxa de molhabilidade.

$$
m=\frac{m}{t}
$$

Onde:

$\mathrm{M}=$ molhabilidade $(\mathrm{g} / \mathrm{s})$;

$\mathrm{m}=$ massa da amostra (mg); e,

$\mathrm{t}=$ tempo $(\mathrm{s})$.

\section{Análise estatística}

Os dados gerados a partir da caracterização físico-química foram submetidos à análise de variância e à comparação entre médias pelo teste de Tukey a 5\% de probabilidade através do programa Statistica, versão 7.7 beta (Silva, \& Azevedo, 2016).

\section{Análise microbiológica}

As determinações microbiológicas foram realizadas no Laboratório de Microbiologia de alimentos da Universidade Federal da Paraíba, Campus III - Bananeiras/PB. Realizaram-se análises microbiológicas no pó do araçá-boi, determinando fungos filamentosos e leveduras, Staphylococcus coagulase positiva, mesófilos aeróbios viáveis, coliformes à $35{ }^{\circ} \mathrm{C}$ e 
termotolerante (em triplicata) e análise de Salmonella spp, segundo recomendações da Instrução Normativa $\mathrm{n}^{\circ}$ 60, de 23 de dezembro de 2019 (BRASIL, 2019) e metodologia de procedimentos pelo Compendium of Methods for the Microbiological Examination of Foods - APHA (Salfinger \& Tortorello, 2015).

\section{Análise das isotermas de adsorção}

As isotermas de adsorção de água do pó de araçá-boi nas temperaturas de 25,30 e $35{ }^{\circ} \mathrm{C}$ foram determinadas de acordo com o método especial indireto estático proposto por Crapiste \& Rotstein, (1982) e adaptado por Lins et al., (2017), utilizando-se o higrômetro Aqualab modelo 3 TE, para medir a atividade de água. Os modelos de Henderson, Oswin, Halsey, GAB e Peleg (Tabela 1) foram ajustados às isotermas de adsorção de água utilizando-se regressão não linear, pelo método Quasi-Newton por meio do programa computacional Statistica 7.7, onde $\mathrm{X}_{\mathrm{e}}=$ teor de água de equilíbrio, $\mathrm{kg} / \mathrm{kg} ; \mathrm{X}_{\mathrm{m}}=$ teor de água na monocamada molecular, $\mathrm{kg} / \mathrm{kg}$; aw = Atividade de água, adimensional; $\mathrm{T}=$ temperatura $\left({ }^{\circ} \mathrm{C}\right) \mathrm{e} \mathrm{a}, \mathrm{b}, \mathrm{c}, \mathrm{k}, \mathrm{k}_{1}, \mathrm{k}_{2},{ }^{\mathrm{n} 1},{ }^{\mathrm{n} 2}=$ constantes das equações.

Tabela 1. Modelos matemáticos para ajustes de isotermas de sorção de água.

\begin{tabular}{|c|c|c|}
\hline Designação do Modelo & Equação & \\
\hline Henderson & $X_{e}=A \cdot \exp (-k \cdot t)$ & (5) \\
\hline Oswin & $\mathrm{X}_{\mathrm{e}}=\mathrm{a}\left(\frac{\mathrm{a}_{\mathrm{w}}}{1-\mathrm{a}_{\mathrm{w}}}\right)^{\mathrm{b}}$ & (6) \\
\hline Halsey & $\mathrm{X}_{\mathrm{e}}=\exp \left(\frac{-\mathrm{A}}{\mathrm{U}_{\mathrm{eq}}^{\mathrm{B}}}\right)$ & (7) \\
\hline GAB (Gugghenheim, Anderson e Boer) & $X_{e}=\left(\frac{X_{m} \cdot C \cdot K \cdot a_{w}}{\left(1-K \cdot a_{w}\right) \cdot\left(1-K \cdot a_{w}+C \cdot K \cdot a_{w}\right)}\right)$ & (8) \\
\hline Peleg & $\mathrm{X}_{\mathrm{e}}=\mathrm{k}_{1} \mathrm{a}_{\mathrm{w}}^{\mathrm{n} 1}+\mathrm{k}_{2} \mathrm{a}_{\mathrm{w}}^{\mathrm{n} 2}$ & (9) \\
\hline
\end{tabular}

Fonte: Autores, seguindo modelos de Henderson, Oswin, Halsey, GAB e Peleg.

Os critérios usados para determinação do melhor ajuste dos modelos aos dados experimentais foram o coeficiente de determinação $\left(\mathrm{R}^{2}\right)$ e o desvio percentual médio $(\mathrm{P})$, calculado pela Equação 10.

$P=\frac{100}{n} \cdot \sum_{i=1}^{n}\left|\frac{\left(X_{\text {exp }}-X_{\text {pre }}\right)}{X_{\text {exp }}}\right|$

Onde:

$\mathrm{P}=$ desvio percentual médio (\%);

$\mathrm{X}_{\text {exp }}=$ valores obtidos experimentalmente;

$\mathrm{X}_{\text {pre }}=$ valores preditos pelo modelo; $\mathrm{e}$,

$\mathrm{n}=$ número de dados experimentais.

\section{Resultados e Discussão}

Na Tabela 2 estão dispostos os resultados referentes a caracterização físico-química das polpas de araçá-boi em pó formuladas com diferentes concentrações de maltodextrina. De início já podemos observar uma diferença significativa entre os valores médios da atividade de água ( $\mathrm{a}_{\mathrm{w}}$ ) e da umidade (Teor de água em \%), onde de acordo com o aumento nas porcentagens de maltodextrina à polpa apresentou redução de aw, assim como no teor de umidade. 
Tabela 2. Caracterização físico-química, de solubilidade e molhabilidade das polpas de araçá-boi em pó com diferentes concentrações de maltodextrina.

\begin{tabular}{|c|c|c|c|}
\hline \multirow{2}{*}{ Parâmetros } & \multicolumn{3}{|c|}{ Concentração MD (\%) } \\
\hline & 14 & 21 & 28 \\
\hline Teor de água (\%) & $7,63 \pm 0,03^{b}$ & $7,88 \pm 0,03^{\mathrm{a}}$ & $7,53 \pm 0,04^{\mathrm{c}}$ \\
\hline Atividade de água $\left(\mathrm{a}_{\mathrm{w}}\right)$ & $0,15 \pm 0,005^{\mathrm{a}}$ & $0,103 \pm 0,02^{b}$ & $0,073 \pm 0,003^{c}$ \\
\hline Sólidos Totais (\%) & $92,37 \pm 0,03^{\mathrm{b}}$ & $92,12 \pm 0,03^{\mathrm{c}}$ & $92,47 \pm 0,04^{\mathrm{a}}$ \\
\hline Acidez titulável (\% ác. cítrico) & $1,59 \pm 0,00^{\mathrm{a}}$ & $1,22 \pm 0,03^{\mathrm{b}}$ & $1,01 \pm 0,01^{\mathrm{c}}$ \\
\hline $\mathrm{pH}$ & $2,82 \pm 0,02^{\mathrm{a}}$ & $2,86 \pm 0,03^{\mathrm{a}}$ & $2,87 \pm 0,02^{\mathrm{a}}$ \\
\hline Sólidos solúveis totais ( $\left.{ }^{\circ} \mathrm{Brix}\right)$ & $8,10 \pm 0,12^{\mathrm{c}}$ & $8,47 \pm 0,06^{\mathrm{b}}$ & $9,10 \pm 0,10^{\mathrm{a}}$ \\
\hline Luminosidade $\left(\mathrm{L}^{*}\right)$ & $73,82 \pm 0,08^{c}$ & $75,94 \pm 0,06^{\mathrm{b}}$ & $76,83 \pm 0,07^{\mathrm{a}}$ \\
\hline Intensidade de vermelho $\left(+\mathrm{a}^{*}\right)$ & $4,35 \pm 0,03^{\mathrm{a}}$ & $3,94 \pm 0,03^{b}$ & $3,87 \pm 0,04^{b}$ \\
\hline Intensidade de amarelo $\left(+b^{*}\right)$ & $26,89 \pm 0,09^{\mathrm{a}}$ & $25,25 \pm 0,28^{b}$ & $24,79 \pm 0,24^{\mathrm{b}}$ \\
\hline Croma $\left(\mathrm{C}^{*}\right)$ & $27,24 \pm 0,09^{\mathrm{a}}$ & $25,55 \pm 0,28^{b}$ & $25,09 \pm 0,24^{\mathrm{b}}$ \\
\hline Ângulo de tonalidade $\left(^{\circ}\right)$ & $80,80 \pm 0,05^{\mathrm{b}}$ & $81,14 \pm 0,15^{\mathrm{a}}$ & $81,12 \pm 0,03^{\mathrm{a}}$ \\
\hline Solubilidade $(\%)$ & $36,12 \pm 1,98^{\mathrm{a}}$ & $32,74 \pm 1,40^{\mathrm{ab}}$ & $29,99 \pm 1,53^{b}$ \\
\hline Molhabilidade (g/s) & $42,67 \pm 0,43^{\mathrm{a}}$ & $11,70 \pm 0,76^{\mathrm{b}}$ & $7,29 \pm 0,36^{\mathrm{c}}$ \\
\hline
\end{tabular}

As médias seguidas da mesma letra minúscula na coluna não diferem entre si ao nível de 5\% de probabilidade pelo teste de Tukey. Fonte: Autores.

O pó de araçá-boi com maior concentração de maltodextrina (28\%) apresentou a menor aw chegando a menos de 0,1 . Todas as formulações demonstraram possuir $\mathrm{a}_{\mathrm{W}}<0,6$, tendo uma média de 0,11 , o que caracterizam produtos de baixa disponibilidade de água utilizada para reações bioquímicas como desenvolvimento de micro-organismos, o que mantem uma estabilidade prolongada a esses produtos, desde que sejam embalados em embalagens que não permitam ou facilitem a absorção de água do ambiente (Rahman, 2019). Esses valores estão próximos dos resultados encontrados por Seerangurayar et al., (2017) que utilizou a Goma Arábica em diferentes concentrações como agente transportador em pós de tâmara, o qual obteve uma variação entre 0,14 e 0,34 na aw

Já para os resultados encontrados para umidade, a qual representa a composição de água em um sistema alimentar como um todo (Hu, 2016), a nossa variação apresentou níveis de 7,63, 7,88 e 7,53\% para as concentrações de 14,21 e $28 \%$ de MD respectivamente. Esse resultado mostra uma semelhança com os números apresentados por Manickavasagan et al., (2015), que ao avaliar pós de tâmaras com adição de maltodextrina, obteve variações de 1,5 a 6,1\% para o teor de umidade. Já Bhusari et al., (2014), ao avaliar pó de tamarindo, também aponta que ao nível em que se aumenta os teores de agentes transportadores de 40 para $50 \%$, o teor de umidade diminui significativamente. Isso pode ser explicado principalmente porque, de acordo com o aumento da quantidade de agentes transportadores (adjuvantes), a higroscopicidade do pó tende a ser reduzida (Seerangurayar et al., 2017).

A adição de maltodextrina à polpa de araçá-boi provocou uma diminuição na acidez titulável em paralelo a porcentagem de maltodextrina adicionada, indo de 1,59 para 1,01\% representada em ácido cítrico, essa diminuição na acidez está em acordo com a queda observada por Iturri et al., (2021), ao avaliar também o pó de araçá-boi, onde mostra valores de 
2,9\% de acidez total, e com relação ${ }^{\circ}$ Brix/acidez de 1,6, mostrando uma baixa gradativa, a qual pode estar relacionada com a adição de adjuntos como MD com alto peso molecular, que possuem alta $\mathrm{Tg}$ que consequentemente diminuem a higroscopicidade do pó.

Com relação aos sólidos solúveis a adição de maltodextrina proporcionou um aumento gradativo de acordo com a quantidade de $\mathrm{MD}$, chegando a um valor de $9,1^{\circ}$ Brix para a concentração de $28 \%$ de MD, esse valor apresenta um aumento de 98,91\% quando relacionado aos valores de ${ }^{\circ}$ Brix encontrados por Garzón et al., (2012) em polpas de araçá-boi sem adição de adjuvantes, com uma média de 4,6 Brix. Também foi observado aumento nos teores de sólidos solúveis em pó de atemoia com aumento de 26,4 para 30,4 ${ }^{\circ}$ Brix (Sousa et al., 2015), e aumento de 7,56 para 9,55 ${ }^{\circ}$ Brix para pó da polpa de cupuaçu (Maciel et al., 2020). Esse aumento na concentração de SST pode está associado a diminuição da umidade (teor de água), e concentração das partículas de açúcares proporcionados pelo alta taxa de Tg incorporadas pela maltodextrina (Barros et al., 2019).

Não foi observado diferença significativa entre as médias do $\mathrm{pH}$ entre as amostras, com todos os valores menores do que 3. No entanto, o pH foi aumentando com adição do adjuvante, isso pode ser explicado pelo fato de que a maltodextrina reduz a concentração dos ácidos orgânicos presentes na amostra, elevando o pH.

Com relação aos resultados da análise colorimétrica, observou-se que a luminosidade (L*) do pó adicionado de maltodextrina apresentou maior valor devido principalmente pela maltodextrina ser branca, deixando a polpa mais clara. Quanto às coordenadas $\mathrm{a}^{*} \mathrm{e} \mathrm{b}^{*}$, observou-se uma diminuição nesses parâmetros no pó contendo maltodextrina. Esses valores de (L*) também são observados por Caparino et al., (2012), que ao avaliar polpa de manga seca por spray, observou as cores mais claras as amostras adicionadas do agente transportador MD. Já esta redução na tonalidade do vermelho e amarelo, pode também está relacionada à diluição da cor, provocada pela presença da maltodextrina (Tonon et al., 2009).

Para as características de solubilidade, observamos uma queda entre as amostras com 14 e $28 \%$ de MD, de 6,13\%. No entanto apesar de ser uma pequena baixa, a solubilidade apresentou uma porcentagem bastante baixa quando comparado com resultados de pó de polpa de manga (Cano-Chauca et al., 2005; Caparino et al., 2012), pó de polpa de graviola (Ceballos et al., 2012); pó de amora preta (Fazaeli et al., 2012) e de pó de polpa de morango (M. I. S. Oliveira et al., 2013) os quais obtiveram um media de $80 \%$ de solubilidade quando utilizados algum agente transportador na composição, sendo assim nossa solubilidade se apresentou muito fora dessas medidas com média de 32,95\% de solubilidade.

A molhabilidade é mediada em gramas por segundo em que mostra o tempo necessário para que a amostra seja absorvida por um líquido (Vissotto et al., 2006). Os nossos resultados apontaram uma queda brusca de 42,67 para 7,29 g/s entre a amostra com 14\% de MD para a amostra com $28 \%$ de MD, caracterizando uma baixa de $82,9 \%$ desse tempo, indicando uma maior molhabilidade com relação a concentração de MD. Oliveira et al., (2015), ao avaliar polpa do cereus jamacaru em pó, também observou uma queda no tempo e aumento na molhabilidade com a adição de maltodextrina. Essa reação de melhor diluição e mais rápida absorção do pó em um liquido é uma questão arbitraria para cada tipo de alimento desenvolvido, e proposito de uso (Medeiros \& Lannes, 2010).

Na Tabela 3 estão os dados da determinação microbiológica da polpa araçá-boi em pó formuladas com diferentes concentrações de maltodextrina. 
Tabela 3. Caracterização microbiológica das polpas de araçá-boi formuladas com diferentes concentrações de maltodextrina.

\begin{tabular}{|c|c|c|c|}
\hline \multirow{2}{*}{ Parâmetros } & \multicolumn{3}{|c|}{ Formulações } \\
\hline & 14 & 21 & 28 \\
\hline Enterobacterias & $<1 \times 10^{2}$ & $<1 \times 10^{2}$ & $<1 \times 10^{2}$ \\
\hline Coliformes Termotolerantes (NMP*/mL) & $<3$ & $<3$ & $<3$ \\
\hline Salmonella spp. $\left({ }^{* *} 25 \mathrm{~g}\right)$ & Ausente & Ausente & Ausente \\
\hline Fungos filamentosos $\left(* * *\right.$ UFC g-10 $\left.0^{-1}\right)$ & $2 \times 10^{1}$ & $1,2 \times 10^{3}$ & $<3$ \\
\hline Staphylococcus aureus (UFC/mL) & $1,7 \times 10^{1}$ & $1 \times 10^{0}$ & $3,5 \times 10^{4}$ \\
\hline
\end{tabular}

*NMP - número mais provável; **A - ausência em 25g; ***UFC - unidade formadora de colônia. Fonte: Autores.

Quanto aos resultados para os microrganismos do grupo dos coliformes termotolerantes, as amostras liofilizadas obedeceram aos padrões bacteriológicos para consumo, cujos valores de contagem padrão foram <3 NMP/g para as três amostras analisadas. A legislação brasileira estabelece para frutas secas e desidratadas limite máximo de $10^{2} \mathrm{NMP} / \mathrm{g}$ para coliformes a $45^{\circ}$.

As polpas de frutas que são secas através do processo de liofilização, que se encontrem em refrigeração adequada, normalmente apresentam características microbiológicas estáveis para o consumo, como os valores encontrados em polpas de acerola, cupuaçu e maracujá comercializadas em Santarém/PA, as quais estavam todas em acordo com a legislação (Sousa et al., 2020).

O resultado das análises microbiológicas do pó do araçá-boi apresentou números satisfatórios para todas as análises liofilizadas associadas ao agente carreador, onde todas obedeceram aos padrões bacteriológicos para consumo. De acordo com os resultados obtidos na polpa de araçá-boi liofilizada, todas as amostras analisadas demonstraram ausência de salmonella spp/25g estando em conformidade com Instrução Normativa nº 60, de 23 de dezembro de 2019 (BRASIL, 2019).

Isso se deve-se principalmente a redução da atividade de água, que torna as condições desfavoráveis para o desenvolvimento da maioria dos microrganismos. Vale também ressaltar que as boas práticas de higiene, manipulação e armazenamento foram satisfatórias durante a elaboração dos produtos contribuindo para a segurança microbiológica dos mesmos.

As formulações das isotermas de adsorção de água foram determinadas na Tabela 4, onde tem-se os parâmetros de ajuste dos modelos de Henderson, Oswin, Halsey, GAB e Peleg ajustados às isotermas de adsorção de água do pó de Araçáboi, nas temperaturas de 25,30 e $35{ }^{\circ} \mathrm{C}$, com seus respectivos coeficientes de determinação $\left(\mathrm{R}^{2}\right)$ e os desvios percentuais médios $(\mathrm{P})$. 
Tabela 4. Parâmetros dos modelos de Henderson, Oswin, Halsey, GAB e Peleg ajustados às isotermas de adsorção de água do pó de araçá-boi, nas temperaturas de 25,30 e $35^{\circ} \mathrm{C}$.

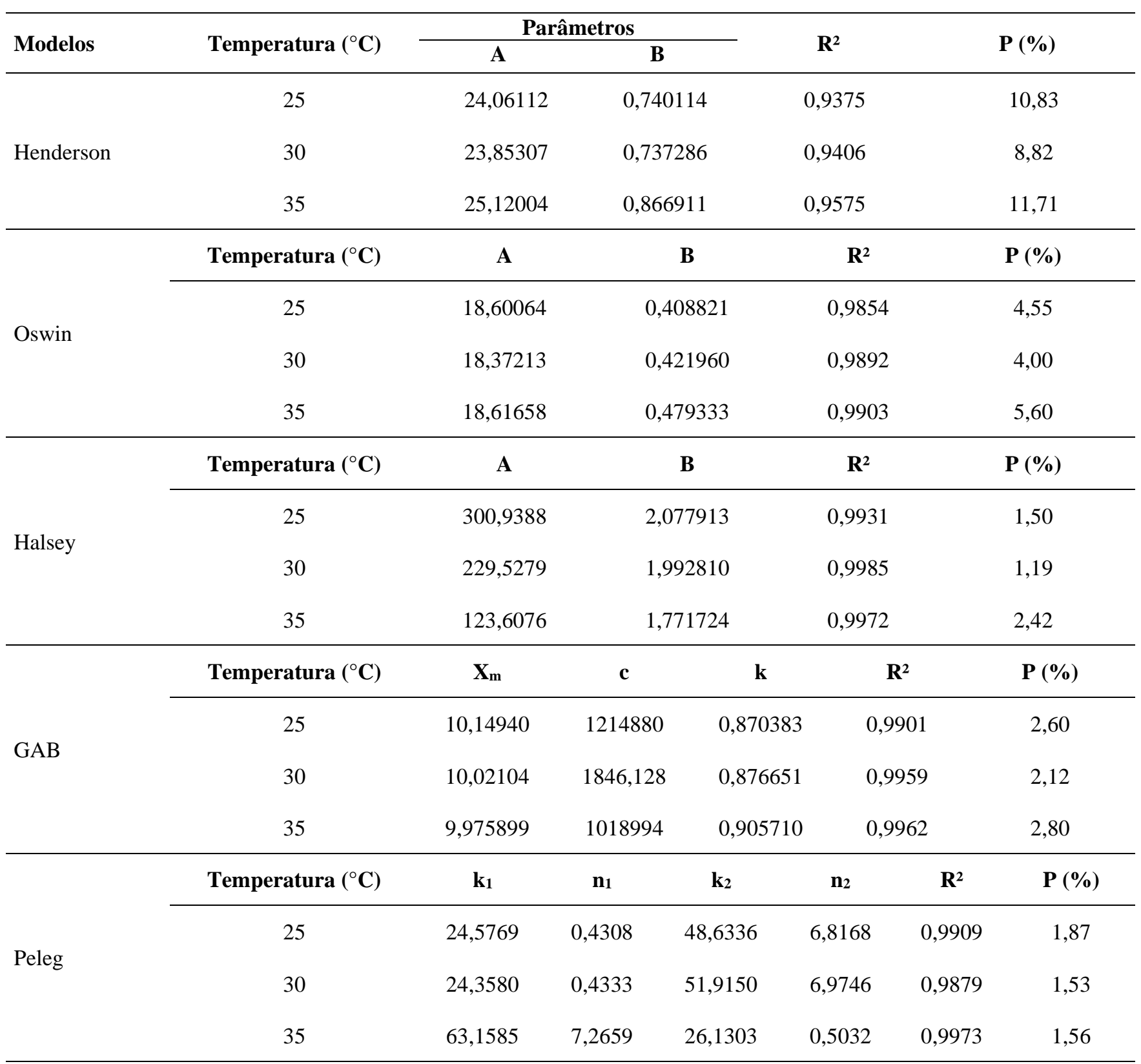

Fonte: Autores.

A partir dos valores do coeficiente de determinação $\left(\mathrm{R}^{2}\right)$ e do desvio percentual médio $(\mathrm{P})$ apresentados na Tabela 4 , verifica-se que, entre os modelos estudados e considerando as temperaturas testadas, os valores de $\mathrm{R}^{2}$ obtidos em GAB, $\mathrm{Halsey}$ e Peleg estão tão próximos entre si que, no pior dos casos, não superam $0,9 \%$ de diferença.

Esses resultados se apresentam semelhante aos valores encontrados por De Sousa Pinto et al., (2019) ao avaliar a polpa em pó de pitaya com porcentagens de 10,20,30, 40, 50 e 100\% de maltodextrina, onde obteve uma média de $\mathrm{R}^{2}$ com 0,97 para todas as formulações.

Considerando os valores do coeficiente de determinação $\left(\mathrm{R}^{2}\right)$ e do erro médio relativo $(\mathrm{P})$, todos os modelos testados descreveram satisfatoriamente as isotermas de adsorção de água do pó de araçá-boi, nas temperaturas analisadas, uma vez que, seus coeficientes ficaram acima de $98 \%$ (com exceção de Henderson), bem como a média do erro relativo inferior a 4,5\%, tendo neste quesito obtido os melhores valores (abaixo da média) nos modelos de Halsey, GAB e Peleg, contudo, a melhor 
representação matemática das isotermas foi obtida pelo modelo de $\mathrm{GAB}$, uma vez que, apresentou os menores valores para $\mathrm{X}_{\mathrm{m}}$ que representa o teor de água na monocamada, sendo portanto, o modelo que melhor explica o ganho de água (PumacahuaRamos et al., 2017).

Esse modelo matemático de GAB também foi descrito por Bezerra et al., (2011) como o melhor ajuste ao avaliar o pó de polpa de manga onde apresentou valores de $\mathrm{R}^{2}$ superiores a 0,96 e com \% inferiores a 10\%. Pumacahua-Ramos et al., (2017) ao avaliar amido de quinoa aponta que o modelo de GAB é ideal para parâmetros que sofrem alterações de temperatura, uma vez que, esse modelo fornece dados de calores de adsorção dos alimentos quando suas constantes $\left(\mathrm{X}_{\mathrm{m}}, \mathrm{C}\right.$ e $\left.\mathrm{K}\right)$ as quais mostram dependência com a temperatura.

O modelo matemático de Guggenheim, Anderson e de Boer (GAB) é um dos modelos mais utilizados em produtos alimentícios, tem como objetivo calcular a massa de água da monocamada e o valor do calor de sorção. Sua equação comporta um parâmetro K a mais, que corresponde a um fator de correção das propriedades da multicamada (Andrade P et al., 2011).

As isotermas de adsorção a 25,30 e $35^{\circ} \mathrm{C}$ do pó de araçá-boi com ajuste pelo modelo de GAB e seus valores do teor de água de equilíbrio com diferentes atividades de água ( $\mathrm{a}_{\mathrm{w}}$ ) podem ser visualizados na Figura 1. As isotermas são obtidas traçando água de equilíbrio de cada pó em função da aw, de forma geral, apresentam forma sigmoidal, em outras palavras, essas isotermas são representadas por curvas que apontam o comportamento da $\mathrm{a}_{\mathrm{W}}$ de um pó, o que acontece com a maioria dos pós alimentícios, a medida em que ele perde (dessorção) e/ou ganha (adsorção) umidade a uma temperatura constante (Cabral de Oliveira et al., 2014).

Essas isotermas podem ser classificadas como curva $\mathrm{S}$, as quais possuem uma inclinação seguida de uma orientação vertical, e podem ser divididas em três partes levando em consideração as condições do meio, a primeira é quando a molécula de soluto é monofuncional e a concentração do adsorbato aumenta até um nível estável (Piccin et al., 2017). No nosso caso observando a Figura 1, esse aumento pode ser percebido estando abaixo de $10 \%$ de diferença entre a aw igual a 0 e aw igual a 0,3. Ainda segundo Piccin et al., (2017), a segunda etapa do comportamento da curva de isoterma apresenta condições satisfatórias quando há uma atração intermolecular moderada, levando a envolvimento vertical entre a camada da molécula adsorvida. Quando observamos a Figura 1, percebemos a variação da $\mathrm{a}_{\mathrm{w}}$ entre 0,3 e 0,6, apresentando um pequeno aumento do teor de água e uma forte modificação da aw. Alcântara et al., (2009) aponta a mesma variação ao avaliar as isotermas de adsorção de pedúnculo de caju em pó.

Isso acontece por que o pó é hidratado por camadas sucessivas da molécula de água, retida por ligações de hidrogênio cada vez mais enfraquecidas, e também por ligações do tipo Van der Waals, em que a participação das moléculas de água nesta fase em reações bioquímicas é muito limitada (Godon \& Willm, 1991). 
Figura 1. Isotermas de adsorção de água nas temperaturas de 25,30 e $35^{\circ} \mathrm{C}$ do pó de Araçá-boi, com ajustes pelo modelo de GAB.

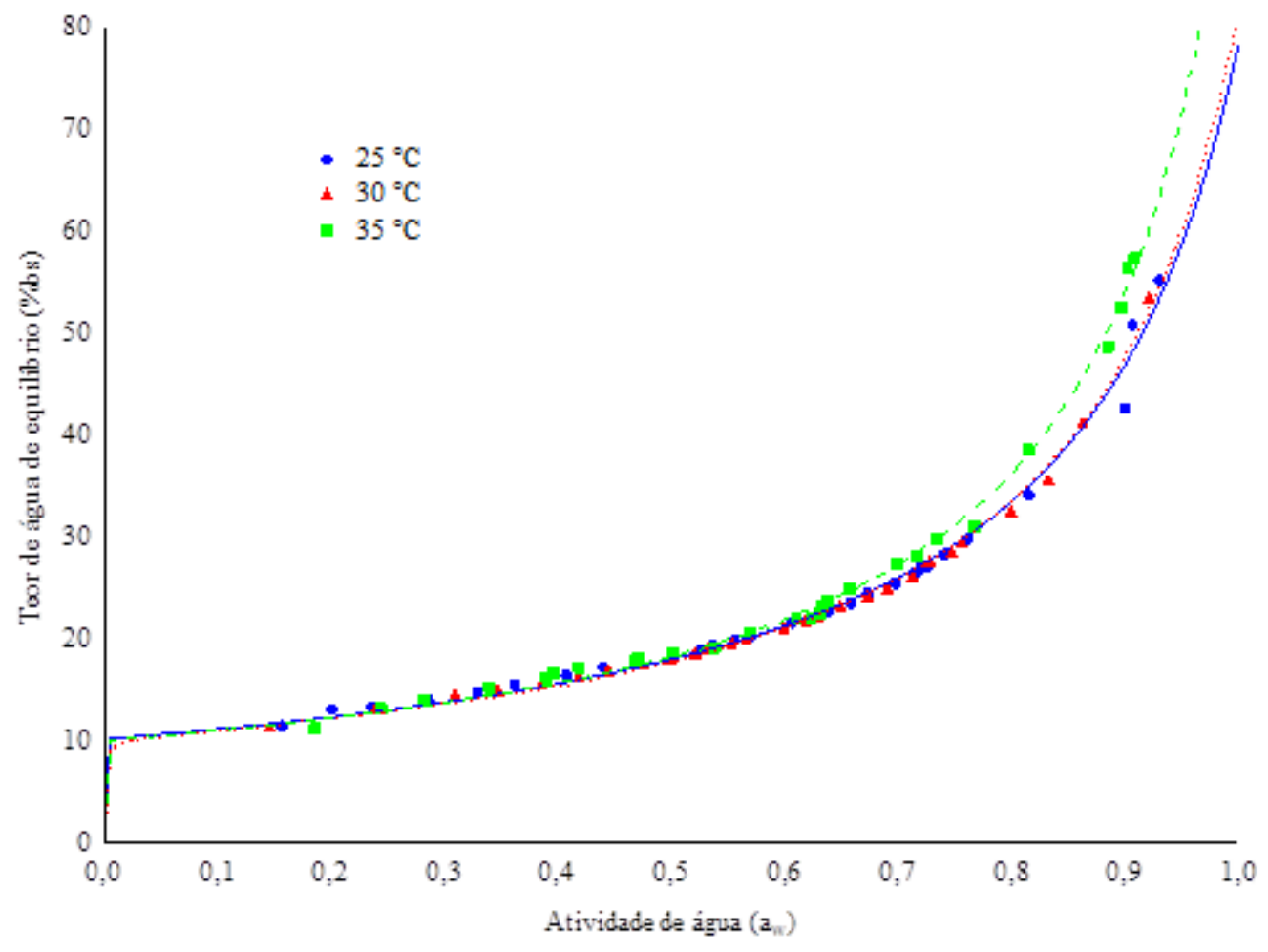

Fonte: Autores, a partir dos dados do modelo matemático de GAB.

Na Figura 1 também podemos notar a terceira parte da isoterma, onde a aw varia de 0,6 a 1, onde se constata um forte aumento do teor de água. De acordo com (Costa et al., (2003), além de Loisel, (1988) e Riganakos et al., (1994), este aumento se dá em consequência da presença de substâncias solúveis e da alta porosidade do substrato. A água presente nesta fase está ligada por forças de natureza capilar ou osmótica, esta água se mantém disponível para as reações bioquímicas.

As isotermas da Figura 1 demonstram a adsorção de água nas temperaturas de 25,30 e $35^{\circ} \mathrm{C}$ do pó de araçá-boi com ajustes pelos modelos de GAB. Apresentando ambas comportamento que Piccin et al., (2017), descreve como que previsto para uma curva de adsorção do tipo S, onde aponta as condições ideais quando há uma forte competição, para locais de substrato, de moléculas do solvente ou outra espécie adsorvida. De acordo com Matos et al., (2014), este comportamento é mais associado à adsorção de alimentos, dadas as características que possuem de absorver grandes concentrações de água com baixa concentração de soluto.

Os modelos de Halsey e de Peleg proporcionaram o melhor ajuste para a adsorção a $35^{\circ} \mathrm{C}$, tal como o de GAB foi o melhor modelo. De acordo com o ajuste obtido para o modelo de GAB, o conteúdo de umidade da camada monomolecular (X0) foi de $0,0582 \mathrm{~kg}$ água/ $\mathrm{kg}$ material seco para o experimento de adsorção a $25^{\circ} \mathrm{C}$ e $0,00845 \mathrm{~kg}$ água $/ \mathrm{kg}$ material seco para a adsorção a $40^{\circ} \mathrm{C}$. Em produtos alimentícios, esse valor é indicativo da estabilidade do alimento frente à maioria das transformações físicas e químicas indesejáveis.

\section{Conclusão}

Com esse estudo sobre o pó do araçá-boi, obtivemos resultados satisfatórios quando relacionado as características físico-químicas, uma vez que, de acordo com aumento da concentração de maltodextrina evidenciamos a manutenção nas quantidades de sólidos totais e do $\mathrm{pH}$, o aumento no brilho das amostras, ou seja maior luminosidade (L*) e principalmente a 
diminuição nos teores de água e aw, proporcionando uma melhor estabilidade do pó.

Para as análises microbiológicas esse estudo apresentou resultados satisfatórios para todas as análises liofilizadas associadas ao agente carreador que obedeceram aos padrões bacteriológicos para consumo.

Além disso podemos evidenciar que a melhor representação matemática das isotermas é obtida pelo modelo de GAB, uma vez que, apresenta os menores valores para $X_{m}$, sendo, portanto, o modelo que melhor explica o ganho de água. Com esses resultados podemos prever melhores condições de conservação, desenvolvimento de embalagens adequadas e conhecimento do teor de umidade de equilíbrio e das características físico-químicas do araçá-boi em pó.

A partir desse estudo sugere-se o levantamento de dados mais aprofundado sobre as a características físico químicas do fruto de araçá-boi, assim como suas características de desenvolvimento de microrganismo, além da avaliação do comportamento higroscópico do pó do araçá-boi obtidas através de outras técnicas de secagem para comparação de resultados.

\section{Referências}

Alcântara, S. R., Almeida, F. de A. C., Silva, F. L. H. da, \& Gomes, J. P. (2009). Isotermas de adsorção do pedúnculo seco do caju. Revista Brasileira de Engenharia Agrícola e Ambiental, 13(1), 81-87. https://doi.org/10.1590/S1415-43662009000100012

Andrade P, R. D., Lemus M, R., \& Pérez C, C. E. (2011). Modelos de isotermas de sorcion para alimentos: Usos y limitaciones. Vitae (Medellín), 325-334.

AOAC. (2016). OFFICIAL METHODS OF ANALYSIS OF AOAC INTERNATIONAL (20 ${ }^{\circ}$ ed, Vol. 20). George W. Latimer Junior. https://www.techstreet.com/standards/official-methods-of-analysis-of-aoac-international-20th-edition-2016?product_id=1937367\#jumps

Baldini, T. F., Neri-Numa, I. A., Do Sacramento, C. K., Schmiele, M., Bolini, H. M. A., Pastore, G. M., \& Bicas, J. L. (2017). Elaboration and Characterization of Apple Nectars Supplemented with Araçá-boi (Eugenia stipitata Mac Vaugh-Myrtaceae). Beverages, 3(4), 59. https://doi.org/10.3390/beverages3040059

Barros, S. L., Santos, N. C., Almeida, R. L. J., Silva, S. do N., Nascimento, A. P. S., Almeida, R. D., Ribeiro, V. H. de A., Silva, W. P., Gomes, J. P., Silva, V. M. de A., Pereira, T. S., Santiago, Â. M., \& Luiz, M. R. (2019). Influence of Pulp, Sugar and Maltodextrin Addiction in the Formulation of Kiwi Jellies With Lemon Grass Tea. Journal of Agricultural Science, 11(15), 125. https://doi.org/10.5539/jas.v11n15p125

Berk, Z. (2018). Freeze drying (lyophilization) and freeze concentration. In Food Process Engineering and Technology (p. 567-581). Elsevier. https://doi.org/10.1016/B978-0-12-812018-7.00023-3

Bezerra, T. S., Costa, J. M. C. da, Afonso, M. R. A., Maia, G. A., \& Clemente, E. (2011). Avaliação físico-química e aplicação de modelos matemáticos na predição do comportamento de polpas de manga desidratadas em pó. Revista Ceres, 58(3), 278-283. https://doi.org/10.1590/S0034-737X2011000300005

Bhusari, S. N., Muzaffar, K., \& Kumar, P. (2014). Effect of carrier agents on physical and microstructural properties of spray dried tamarind pulp powder. Powder Technology, 266, 354-364. https://doi.org/10.1016/j.powtec.2014.06.038

Botrel, D. A., Rodrigues, I. C. B., Souza, H. J. B. de, \& Fernandes, R. V. de B. (2016). Application of inulin in thin-layer drying process of araticum (Annona crassiflora) pulp. LWT - Food Science and Technology, 69, 32-39. https://doi.org/10.1016/j.1wt.2016.01.018

BRASIL. (2019, dezembro 23). ANVISA - Padrões microbiológicos para alimentos prontos para oferta ao consumidor. INSTRUÇÃO NORMATIVA N ${ }^{\circ}$ 60, DE 23 DE DEZEMBRO DE 2019. https://www.cevs.rs.gov.br/upload/arquivos/202004/17093620-in-anvisa-60-2019.pdf

Cabral de Oliveira, D. E., Resende, O., Cândido Campos, R., \& Rodrigues Donadon, J. (2014). Obtenção e modelagem das isotermas de dessorção e do calor isostérico para sementes de arroz em casca. Científica, 42(3), 203. https://doi.org/10.15361/1984-5529.2014v42n3p203-210

Calvi, G. P., Aud, F. F., Ferraz, I. D. K., Pritchard, H. W., \& Kranner, I. (2017). Analyses of several seed viability markers in individual recalcitrant seeds of Eugenia stipitata McVaugh with totipotent germination. Plant Biology, 19(1), 6-13. https://doi.org/10.1111/plb.12466

Cano-Chauca, M., Stringheta, P. C., Ramos, A. M., \& Cal-Vidal, J. (2005). Effect of the carriers on the microstructure of mango powder obtained by spray drying and its functional characterization. Innovative Food Science \& Emerging Technologies, 6(4), 420-428. https://doi.org/10.1016/j.ifset.2005.05.003

Caparino, O. A., Tang, J., Nindo, C. I., Sablani, S. S., Powers, J. R., \& Fellman, J. K. (2012). Effect of drying methods on the physical properties and microstructures of mango (Philippine ‘Carabao' var.) powder. Journal of Food Engineering, 111(1), 135-148. https://doi.org/10.1016/j.jfoodeng.2012.01.010

Cavalcante, C. E. B., Rodrigues, S., Afonso, M. R. A., Costa, J. M. C., Cavalcante, C. E. B., Rodrigues, S., Afonso, M. R. A., \& Costa, J. M. C. (2018). Comportamento higroscópico da polpa de graviola em pó obtida por secagem em spray dryer. Brazilian Journal of Food Technology, 21. https://doi.org/10.1590/1981-6723.12117

Ceballos, A. M., Giraldo, G. I., \& Orrego, C. E. (2012). Effect of freezing rate on quality parameters of freeze dried soursop fruit pulp. Journal of Food Engineering, 111(2), 360-365. https://doi.org/10.1016/j.jfoodeng.2012.02.010

Çopur, Ö. U., İncedayı, B., \& Karabacak, A. Ö. (2019). Technology and Nutritional Value of Powdered Drinks. In Production and Management of Beverages (p. 47-83). Elsevier. https://doi.org/10.1016/B978-0-12-815260-7.00002-X 
Costa, J. M. C., Medeiros, M. de F. D., \& da Mata, A. L. M. (2003). A comparative study on the adsorption isotherms of beetroot (Beta vulgaris, L.), pumpkin (Cucurbita moschata) and carrot (Daucus carota) powder form obtained by airbed drying process. Revista Ciência Agronômica.

Costa, W. K., Oliveira, J. R. S. de, Oliveira, A. M. de, Santos, I. B. da S., Cunha, R. X. da, Freitas, A. F. S. de, Silva, J. W. L. M. da, Silva, V. B. G., Aguiar, J. C. R. de O. F. de, Silva, A. G. da, Navarro, D. M. do A. F., Lima, V. L. de M., \& Silva, M. V. da. (2020). Essential oil from Eugenia stipitata McVaugh leaves has antinociceptive, anti-inflammatory and antipyretic activities without showing toxicity in mice. Industrial Crops and Products, 144, 112059. https://doi.org/10.1016/j.indcrop.2019.112059

Crapiste, G. H., \& Rotstein, E. (1982). Prediction of Sorptional Equilibrium Data for Starch-Containing Foodstuffs. Journal of Food Science, 47(5), 15011507. https://doi.org/10.1111/j.1365-2621.1982.tb04970.x

De Sousa Pinto, E. M., Guerreiro de Faria, L. J., Pereira Costa, W. J., De Sousa Araújo Neto, C. A., Rocha Pereira, R., \& Fonseca da Silva, C. L. (2019). Estudo higroscópico da polpa em pó do fruto da pitaya (Hylocereus costaricencis) em diferentes concentrações de maltodextrina. Revista de la Facultad de Agronomía, 118(2), 029. https://doi.org/10.24215/16699513e029

De Souza, R. S., Silva, S. S. e, Loss, R. A., Souza, R. D. S., \& Guedes, S. F. (2018). AVALIAÇÃo FÍSICO-QUÍMICA DO FRUTO ARAÇÁ-BOI (Eugenia stipitata MacVaugh) CULTIVADO NA MESORREGIÃO DO SUDOESTE MATO-GROSSENSE. Revista Destaques Acadêmicos, 10(3). https://doi.org/10.22410/issn.2176-3070.v10i3a2018.1948

Djendoubi Mrad, N., Bonazzi, C., Boudhrioua, N., Kechaou, N., \& Courtois, F. (2012). Influence of sugar composition on water sorption isotherms and on glass transition in apricots. Journal of Food Engineering, 111(2), 403-411. https://doi.org/10.1016/j.jfoodeng.2012.02.001

Eastman, J. E., \& Moore, C. O. (1984). Cold-water-soluble granular starch for gelled food compositions (United States Patent $\mathrm{N}^{\circ}$ US4465702A). https://patents.google.com/patent/US4465702A/en

Fazaeli, M., Emam-Djomeh, Z., Kalbasi Ashtari, A., \& Omid, M. (2012). Effect of spray drying conditions and feed composition on the physical properties of black mulberry juice powder. Food and Bioproducts Processing, 90(4), 667-675. https://doi.org/10.1016/j.fbp.2012.04.006

Fongin, S., Alvino Granados, A. E., Harnkarnsujarit, N., Hagura, Y., \& Kawai, K. (2019). Effects of maltodextrin and pulp on the water sorption, glass transition, and caking properties of freeze-dried mango powder. Journal of Food Engineering, 247, 95-103. https://doi.org/10.1016/j.jfoodeng.2018.11.027

Freudig, B., Hogekamp, S., \& Schubert, H. (1999). Dispersion of powders in liquids in a stirred vessel. Chemical Engineering and Processing: Process Intensification, 38(4), 525-532. https://doi.org/10.1016/S0255-2701(99)00049-5

Garzón, G. A., Narváez-Cuenca, C.-E., Kopec, R. E., Barry, A. M., Riedl, K. M., \& Schwartz, S. J. (2012). Determination of Carotenoids, Total Phenolic Content, and Antioxidant Activity of Arazá (Eugenia stipitata McVaugh), an Amazonian Fruit. Journal of Agricultural and Food Chemistry, 60(18), 47094717. https://doi.org/10.1021/jf205347f

Godon, B., \& Willm, C. (1991). Les industries de premiere transformation des cereales. https://agris.fao.org/agris-search/search.do?recordID=XF2015019403

Gonçalves, A. E. D. S. S., Lajolo, F. M., \& Genovese, M. I. (2010). Chemical Composition and Antioxidant/Antidiabetic Potential of Brazilian Native Fruits and Commercial Frozen Pulps. Journal of Agricultural and Food Chemistry, 58(8), 4666-4674. https://doi.org/10.1021/jf903875u

Hamid, Thakur, N. S., Thakur, A., \& Kumar, P. (2020). Effect of different drying modes on phenolics and antioxidant potential of different parts of wild pomegranate fruits. Scientia Horticulturae, 274, 109656. https://doi.org/10.1016/j.scienta.2020.109656

Hu, M. (2016). Oxidative Stability and Shelf Life of Low-Moisture Foods. In Oxidative Stability and Shelf Life of Foods Containing Oils and Fats (p. 313371). Elsevier. https://doi.org/10.1016/B978-1-63067-056-6.00009-4

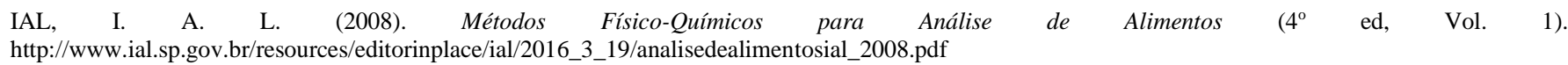

Iturri, M. S., Calado, C. M. B., \& Prentice, C. (2021). Microparticles of Eugenia stipitata pulp obtained by spray-drying guided by DSC: An analysis of bioactivity and in vitro gastrointestinal digestion. Food Chemistry, 334, 127557. https://doi.org/10.1016/j.foodchem.2020.127557

Karim, M. A., Rahman, M. M., Pham, N. D., \& Fawzia, S. (2018). Food Microstructure as affected by processing and its effect on quality and stability. In Food Microstructure and Its Relationship with Quality and Stability (p. 43-57). Elsevier. https://doi.org/10.1016/B978-0-08-100764-8.00003-4

Lins, A. D. F., Rocha, A. P. T., Gomes, J. P., Feitosa, R. M., Araujo, G. T., \& Santos, D. da C. (2017). Adsorption isotherms of the red mombin powder produced in spouted bed dryer. Revista Brasileira de Engenharia Agrícola e Ambiental, 21(8), 562-567. https://doi.org/10.1590/18071929/agriambi.v21n8p562-567

Loisel, C. (1988). Contribution à l'étude des propriétés rhéologiques de quelques poudres alimentaires: Influence des conditions hygrométriques [These de doctorat, Nantes]. https://www.theses.fr/1988NANT2035

Maciel, R. M. G., Lima, S. B. de, Costa, J. M. C. da, \& Afonso, M. R. A. (2020). Influência da maltodextrina nas propriedades de escoamento do pó da polpa de cupuaçu / Influence of maltodextrin on the flow properties of the cupuaçu pulp powder. Brazilian Journal of Development, 6(2), 5829-5839. https://doi.org/10.34117/bjdv6n2-039

Manickavasagan, A., Thangavel, K., Dev, S. R. S., Delfiya, D. S. A., Nambi, E., Orsat, V., \& Raghavan, G. S. V. (2015). Physicochemical Characteristics of Date Powder Produced in a Pilot-Scale Spray Dryer. Drying Technology, 33(9), 1114-1123. https://doi.org/10.1080/07373937.2015.1014045

Matos, E. N. V., Sampaio, R. M., \& Paula, M. D. L. de. (2014). Determinação Das Isotermas De Adsorção E Do Calor De Sorção De Farinha De Babaçu Comercial. Cadernos de Pesquisa, 21. https://doi.org/10.18764/2178-2229.v21.n.especial.p.21-29

Medeiros, M. L., \& Lannes, S. C. da S. (2010). Propriedades físicas de substitutos do cacau. Food Science and Technology, 30, $243-253$. https://doi.org/10.1590/S0101-20612010000500037 
Miletić, N., Popović, B., Mitrović, O., Kandić, M., \& Leposavić, A. (2014). Phenolic compounds and antioxidant capacity of dried and candied fruits commonly consumed in Serbia\&nbsp; Czech Journal of Food Sciences, 32(No. 4), 360-398. https://doi.org/10.17221/166/2013-CJFS

Najafabadi, N. S., Sahari, M. A., Barzegar, M., \& Esfahani, Z. H. (2017). Effect of gamma irradiation on some physicochemical properties and bioactive compounds of jujube (Ziziphus jujuba var vulgaris) fruit. Radiation Physics and Chemistry, 130, 62-68. https://doi.org/10.1016/j.radphyschem.2016.07.002

Neri-Numa, I. A., Carvalho-Silva, L. B., Morales, J. P., Malta, L. G., Muramoto, M. T., Ferreira, J. E. M., de Carvalho, J. E., Ruiz, A. L. T. G., Maróstica Junior, M. R., \& Pastore, G. M. (2013). Evaluation of the antioxidant, antiproliferative and antimutagenic potential of araçá-boi fruit (Eugenia stipitata Mc Vaugh-Myrtaceae) of the Brazilian Amazon Forest. Food Research International, 50(1), 70-76. https://doi.org/10.1016/j.foodres.2012.09.032

Neves, L. C., Tosin, J. M., Benedette, R. M., \& Cisneros-Zevallos, L. (2015). Post-harvest nutraceutical behaviour during ripening and senescence of 8 highly perishable fruit species from the Northern Brazilian Amazon region. Food Chemistry, 174, 188-196. https://doi.org/10.1016/j.foodchem.2014.10.111

Oliveira, A. da S., Figueirêdo, R. M. F. de, Queiroz, A. J. de M., Brito, J. G. de, Oliveira, A. da S., Figueirêdo, R. M. F. de, Queiroz, A. J. de M., \& Brito, J. G. de. (2015). Estabilidade da polpa do Cereus jamacaru em pó durante o armazenamento. Revista Brasileira de Engenharia Agrícola e Ambiental, 19(2), 147153. https://doi.org/10.1590/1807-1929/agriambi.v19n2p147-153

Oliveira, G. S., Costa, J. M. C. da, \& Afonso, M. R. A. (2014). Caracterização e comportamento higroscópico do pó da polpa de cajá liofilizada. Revista Brasileira de Engenharia Agrícola e Ambiental, 18(10), 1059-1064. https://doi.org/10.1590/1807-1929/agriambi.v18n10p1059-1064

Oliveira, M. I. S., Tonon, R. V., Nogueira, R. I., \& Cabral, L. M. C. (2013). Estabilidade da polpa de morango atomizada utilizando diferentes agentes carreadores. Brazilian Journal of Food Technology, 16(4), 310-318. https://doi.org/10.1590/S1981-67232013005000037

Orqueda, M. E., Zampini, I. C., Torres, S., Alberto, M. R., Pino Ramos, L. L., Schmeda-Hirschmann, G., \& Isla, M. I. (2017). Chemical and functional characterization of skin, pulp and seed powder from the Argentine native fruit mistol (Ziziphus mistol). Effects of phenolic fractions on key enzymes involved in metabolic syndrome and oxidative stress. Journal of Functional Foods, 37, 531-540. https://doi.org/10.1016/j.jff.2017.08.020

Palzer, S., \& Sommer, K. (2011). Caking of Water-Soluble Amorphous and Crystalline Food Powders. In J. M. Aguilera, R. Simpson, J. Welti-Chanes, D. Bermudez-Aguirre, \& G. Barbosa-Canovas (Orgs.), Food Engineering Interfaces (p. 491-514). Springer. https://doi.org/10.1007/978-1-4419-7475-4_19

Pereira, A. S., Shitsuka, D. M., Parreira, F. J., \& Shitsuka, R. (2018). Metodologia Da Pesquisa Científica. https://repositorio.ufsm.br/bitstream/handle/1/15824/Lic_Computacao_Metodologia-Pesquisa-Cientifica.pdf?sequence=1

Piccin, J. S., Cadaval, T. R. S., de Pinto, L. A. A., \& Dotto, G. L. (2017). Adsorption Isotherms in Liquid Phase: Experimental, Modeling, and Interpretations. In A. Bonilla-Petriciolet, D. I. Mendoza-Castillo, \& H. E. Reynel-Ávila (Orgs.), Adsorption Processes for Water Treatment and Purification (p. 19-51). Springer International Publishing. https://doi.org/10.1007/978-3-319-58136-1_2

Pumacahua-Ramos, A., Limaylla-Guerrero, K. M., Telis-Romero, J., \& Lopes-Filho, J. F. (2017). ISOTERMAS Y CALOR ISOSTÉRICO DE ADSORCIÓN DE AGUA DE ALMIDÓN DE QUINUA. Biotecnología en el Sector Agropecuario y Agroindustrial, 15(1), 95-104. https://doi.org/10.18684/BSAA(15)95104

Rahman, M. S. (2019). Water Activity and Glass Transition of Foods. In Reference Module in Food Science (p. B9780081005965212000). Elsevier. https://doi.org/10.1016/B978-0-08-100596-5.21184-0

Riganakos, K. A., Demertzis, P. G., \& Kontominas, M. G. (1994). Water Sorption by Wheat and Soy Flour: Comparison of Three Methods. Journal of Cereal Science, 20(1), 101-106. https://doi.org/10.1006/jcrs.1994.1050

Salfinger, Y., \& Tortorello, M. L. (Orgs.). (2015). Compendium of Methods for the Microbiological Examination of Foods. American Public Health Association. https://doi.org/10.2105/MBEF.0222

Seerangurayar, T., Manickavasagan, A., Al-Ismaili, A. M., \& Al-Mulla, Y. A. (2017). Effect of carrier agents on flowability and microstructural properties of foam-mat freeze dried date powder. Journal of Food Engineering, 215, 33-43. https://doi.org/10.1016/j.jfoodeng.2017.07.016

Silva, F. de A. S., \& Azevedo, C. A. V. de. (2016). The Assistat Software Version 7.7 and its use in the analysis of experimental data. African Journal of Agricultural Research, 11(39), 3733-3740. https://doi.org/10.5897/AJAR2016.11522

Soares, J. C., Rosalen, P. L., Lazarini, J. G., Massarioli, A. P., da Silva, C. F., Nani, B. D., Franchin, M., \& de Alencar, S. M. (2019). Comprehensive characterization of bioactive phenols from new Brazilian superfruits by LC-ESI-QTOF-MS, and their ROS and RNS scavenging effects and anti-inflammatory activity. Food Chemistry, 281, 178-188. https://doi.org/10.1016/j.foodchem.2018.12.106

Sousa, Figueirêdo, R. M. F. D., Queiroz, A. J. D. M., Fernandes, T. K. D. S., Sousa, K. D. S. M. D., Figueirêdo, R. M. F. D., Queiroz, A. J. D. M., \& Fernandes, T. K. D. S. (2015). Produção E Caracterização Da Polpa De Atemoia Em Pó. Revista Brasileira de Fruticultura, 37(3), 718-728. https://doi.org/10.1590/0100-2945-135/14

Sousa, Y. A., Borges, M. A., Viana, A. F. da S., Dias, A. L., Sousa, J. J. V. de, Silva, B. A. da, Silva, S. K. R. da, Aguiar, F. S. de, Sousa, Y. A., Borges, M. A., Viana, A. F. da S., Dias, A. L., Sousa, J. J. V. de, Silva, B. A. da, Silva, S. K. R. da, \& Aguiar, F. S. de. (2020). Avaliação físico-química e microbiológica de polpas de frutas congeladas comercializadas em Santarém-PA. Brazilian Journal of Food Technology, 23. https://doi.org/10.1590/1981-6723.08518

Sun, Y.-F., Liang, Z.-S., Shan, C.-J., Viernstein, H., \& Unger, F. (2011). Comprehensive evaluation of natural antioxidants and antioxidant potentials in Ziziphus jujuba Mill. Var. Spinosa (Bunge) Hu ex H. F. Chou fruits based on geographical origin by TOPSIS method. Food Chemistry, 124(4), 1612-1619. https://doi.org/10.1016/j.foodchem.2010.08.026

Tonon, R. V., Brabet, C., \& Hubinger, M. D. (2009). Influência da temperatura do ar de secagem e da concentração de agente carreador sobre as propriedades físico-químicas do suco de açaí em pó. Food Science and Technology, 29(2), 444-450. https://doi.org/10.1590/S0101-20612009000200034 
Research, Society and Development, v. 10, n. 9, e24810916515, 2021

(CC BY 4.0) | ISSN 2525-3409 | DOI: http://dx.doi.org/10.33448/rsd-v10i9.16515

Vissotto, F. Z., Jorge, L. C., Makita, G. T., Rodrigues, M. I., \& Menegalli, F. C. (2010). Influence of the process parameters and sugar granulometry on cocoa beverage powder steam agglomeration. Journal of Food Engineering, 97(3), 283-291. https://doi.org/10.1016/j.jfoodeng.2009.10.013

Vissotto, Montenegro, F. M., Santos, J. M. dos, \& Oliveira, S. J. R. de. (2006). Avaliação da influência dos processos de lecitinação e de aglomeração nas propriedades físicas de achocolatado em pó. Ciência e Tecnologia de Alimentos, 26(3), 666-671. https://doi.org/10.1590/S0101-20612006000300028 